\title{
轻质复合材料飞行器仪器支架选择性激光 烧结成形与性能研究*
}

\author{
晏梦雪 $^{1}$ 田小永 ${ }^{1}$ 彭 刚 ${ }^{1}$ 李涤尘 $^{1}$ 姚瑞娟 $^{2}$ \\ 张 薇 ${ }^{2}$ 白 锐 ${ }^{3}$ 孟伟杰 $^{3}$
}

(1. 西安交通大学机械制造系统工程国家重点实验室 西安 710049;

2. 北京宇航系统工程研究所 北京 100076 ;

3. 达索析统(上海)信息技术有限公司 上海 200120)

摘要: 针对目前飞行器仪器支架对轻量化、快速开发及结构优化的要求, 提出使用选择性激光烧结(SLS)工艺制备轻质复合 材料仪器支架, 并对其性能进行了综合评估。根据飞行器仪器支架的使用环境, 首先对 SLS 制备短切碳纤维增强尼龙复合材 料(CF/PA12)进行了工艺优化并详细研究了复合材料的力学性能、热性能及动态热力学性能, 试验结果表明, SLS 制备的 CF/PA12 复合材料的拉伸强度达到 $63.8 \mathrm{MPa}$, 弹性模量为 $6.5 \mathrm{GPa}$, 弯曲强度达到 $118.06 \mathrm{MPa}$, 体积密度仅为 $1.03 \mathrm{~g} / \mathrm{cm}^{3}$, 尤 其是其损耗因子在 $0.03 \sim 0.06$ 之间，远远大于金属材料，具有更好的减振性能，并对其装配形式进行了评估。最后，以一款 火箭仪器支架为例, 通过拓扑优化, 实现支架进一步减重 $40 \%$, 且都通过了载荷试验 $(2000 \mathrm{~N})$, 从而证明了 SLS 制备复合材 料在航空航天领域制件轻量化、快速开发及结构优化方面具有明显的技术优势和一定的应用潜力。

关键词: 仪器支架; 尼龙复合材料; 选择性激光烧结; 增材制造; 拓扑优化

中图分类号: TH64; TB332

\section{Performance Study of Lightweight Composites Equipment Section Support Fabricated by Selective Laser Sintering}

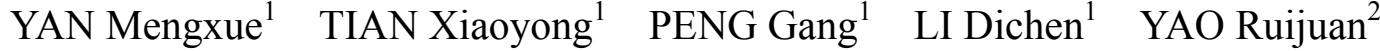 \\ ZHANG Wei ${ }^{2}$ BAI Rui ${ }^{3}$ MENG Weijie ${ }^{3}$
}

(1. State Key Laboratory of Manufacturing Systems Engineering, Xi’an Jiaotong University, Xi'an 710049;

2. China Academy of Launch Vehicle Technology, Beijing 100076;

3. Dassault Systems (Shanghai) Information Technology Co., Ltd. Shanghai 200120)

\begin{abstract}
A novel method to prepare equipment section support for flight vehicle is proposed by using lightweight thermoplastic composites prepared by selective laser sintering (SLS), in order to meet the requirements of lightweight, rapid development and structure optimization in equipment section support. Considering the special service environment of equipment section support, the process parameter optimization for preparing the short carbon fiber reinforced PA12 composites by SLS is carried out and the mechanical property, thermal behavior and dynamic mechanical property of the carbon fiber reinforced nylon 12 composites prepared by SLS are studied, and the test results show that the tensile strength is about $63.8 \mathrm{MPa}$ with an elastic modulus of $6.5 \mathrm{GPa}$, the flexural strength reached to $118.06 \mathrm{MPa}$ and the damping factor is $0.03-0.06$, which is much higher than metal material, indicating a better damping performance. The fracture morphology of composites shows massive pores and its bulk density is only about 1.03 $\mathrm{g} / \mathrm{cm}^{3}$, which is far lower than metal material. Meanwhile, several assemble methods are used for the composites prepared by SLS, and the pulling-out force and failure behavior are assessed. At last, an equipment section support in rocket and the optimized structural support are prepared by SLS, and the weight of the optimized structural support is only $60 \%$ of the original. The results of load experiment shows these two supports both can withstand the load of $2000 \mathrm{~N}$ without destruction and clear deformation, which
\end{abstract}

* 国家高技术研究发展计划(863 计划 2015AA042503)和重型运载火箭工 程塑料仪器支架研制及支架试验技术研究资助项目。20180716 收到初 稿, 20181229 收到修改稿 
demonstrated the feasibility and the potentials in the aerospace field of proposed method, as the technical advantage of SLS in the lightweight, rapid development and structure optimization.

Key words: equipment section support; carbon fiber reinforced nylon12; selective laser sintering; additive manufacturing; topology optimization

\section{0 前言}

仪器支架作为飞行器( 火箭、导弹等)承载导航 设备及控制系统等重要电气系统的安装平台, 其结 构性能的好坏直接影响到仪器设备的正常使用, 尤 其是结构强度和振动性能。同时, 仪器支架的轻量 化对于飞行器的减重也尤为重要, 与普通金属材料 相比, 复合材料具有更高的比强度和比刚度, 已被 应用于火箭仪器舱及仪器支架的制备, 2002 年, 俄 罗斯 PROTON-M 运载火箭 ${ }^{[1]}$ 首次使用了碳纤维增 强复合材料制造仪器舱外舱体的支撑结构, 相比于 铝合金材料, 减重可达 $40 \%$ 。此外, 在航天工程领 域, 振动问题及材料的减振性能也一直是备受关注, 相对金属材料, 高分子材料及其复合材料作为一种 典型的粘弹性材料, 其材料损耗因子一般在 0.005 左右 ${ }^{[2]}$, 而金属材料的损耗因子则远远小于高分子 材料, 其值一般在 $0.0001 \sim 0.0006^{[3]}$, 这意味着高 分子材料制件在振动环境中, 趋向于将部分振动能 转换为内能, 从而具有一定的减振功能 ${ }^{[4]}$ 。

此外, 火箭及其他飞行器的仪器舱内还存在着 设备多、安装空间小等限制, 这就对舱内支架的结 构设计及布置提出了更高的要求。然而, 目前飞行 器的仪器支架一般采用铝、镁合金等金属材料进行 制备, 同时在支架与仪器之间加入阻尼减震器以降 低振动 ${ }^{[5]}$, 这就进一步造成仪器支架的布置难度。

与此同时, 金属仪器支架的成型一般需要开发配套 模具, 这就造成产品的开发周期较长且不便于支架 的结构调整。

选择性激光烧结(Selective laser sintering, SLS) 是增材制造技术中广泛使用的一种制造方法, 可用 于高分子材料及其复合材料的直接成形, 具有制备 速度快、无需模具、制件强度较好、可制备任意复 杂结构的零件等优点 ${ }^{[6-7]}$, 这就为产品的结构优化及 快速开发提供了可能性; 对于半结晶高分子材料, 如尼龙、PEEK 等, 其制件强度基本达到注塑件的 强度, 可直接用作结构件 ${ }^{[8-9]}$ 。目前, 随着相关研究 的不断深入, SLS 制备的高分子复合材料种类及其 应用都在不断拓展 ${ }^{[10-12]}$, 但是, SLS 制备的复合材 料制件对于复杂力学和温度环境的适应性研究尚未 系统开展。
因此, 本文针对飞行器仪器支架的受力环境, 对 SLS 制备的短切碳纤维增强尼龙复合材料 (CF/PA12)的力学性能开展了研究, 包括基础力学性 能、高温力学性能、动态机械性能等; 同时, 对其 装配方式与性能也展开了研究, 评估了多种装配方 式的螺纹拉脱力和不同的螺纹孔装配体的破坏形 式; 最后, 以一款火箭仪器支架为例进行了样件制 备, 并对其进行了拓扑结构优化设计, 采用 SLS 对 优化后的支架进行了制造, 通过载荷试验对支架的 承载能力进行评估分析。

\section{1 试验方案与性能评估}

\section{1 选择性激光烧结成形工艺与材料}

选择性激光烧结以高分子粉末作为原材料, 通 过激光烧结区域的控制逐层完成实体的自由成形, 如图 1 所示。由于粉末材料的自身优势, 即易与其 他材料进行混合, 从而可以有目的设计材料配方, 以提高复合材料在某一方面的性能, 本文采用在尼 龙 12 粉末中添加短切碳纤维以提高复合材料的机 械性能与耐热性, 其中碳纤维的质量分数为 $40 \%$, 复合粉末的微观结构如图 2a 所示, 粒径分布如图 $2 \mathrm{~b}$ 所示, 从图中可以看出, 尼龙颗粒呈现较规则的 球形，粒径集中在 $10 \sim 100 \mu \mathrm{m}$ 之间，且 95\%尼龙 颗粒粒径小于 $120 \mu \mathrm{m}$, 而碳纤维的长度主要分布在 $200 \sim 700 \mu \mathrm{m}$ 之内。

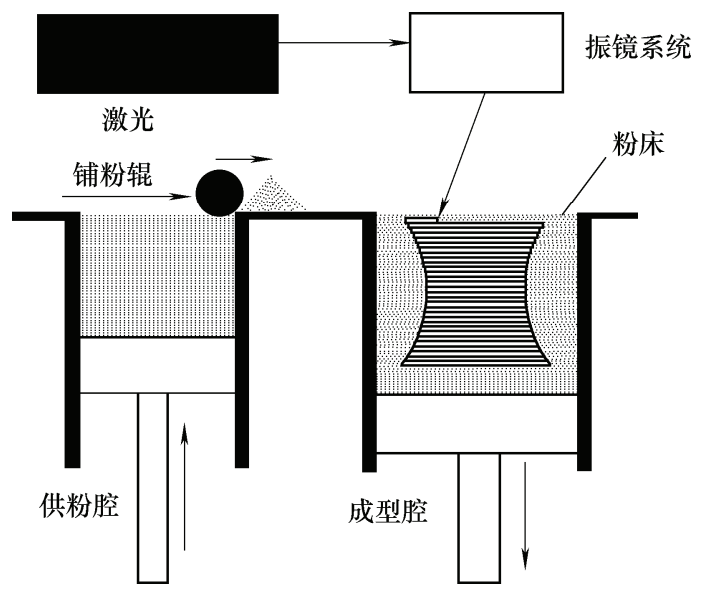

图 1 SLS 工艺示意图

试验中使用西安交通大学自制的选择性激光烧 结成形设备, 对标准试样及支架进行制备。该选择 


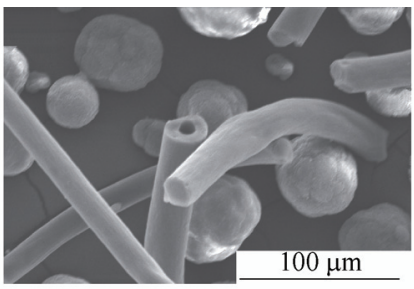

(a)

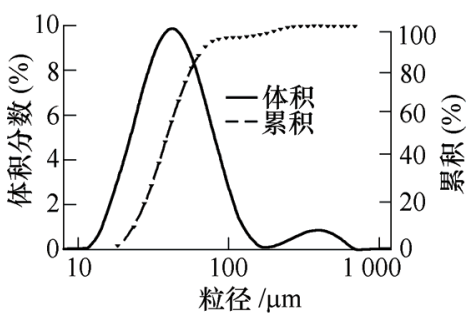

(b)

图 2 CF/PA12 复合粉末微观结构及粒径分布

性结成形机的成形腔体尺寸为 $300 \mathrm{~mm} \times 300 \mathrm{~mm} \times$ $400 \mathrm{~mm}$; 配备有一台二氧化碳激光器(波长 $10.6 \mu \mathrm{m}$ ), 经扩束镜、振镜等光路系统后, 可完成给定路径的 激光扫描, 最大功率为 $60 \mathrm{~W}$, 光斑直径为 $0.3 \mathrm{~mm}$; 成形腔内采用红外灯管对粉末床进行预热, 同时, 还配备有速度可调的铺粉辊以保证复合粉末能够形 成平整的粉末床。由于选择性激光烧结的工艺特点, 预热温度、激光扫描速度、扫描间距、激光功率及 分层厚度等, 都会对制件的性能产生影响, 其中预 热温度的选择一般根据粉末的熔融及重结晶温度进 行确定, 根据相关测试结果, 预热温度设置为 $150{ }^{\circ} \mathrm{C}$; 同时, 固定激光扫描速度 $3000 \mathrm{~mm} / \mathrm{s}$, 扫 描间距 $0.12 \mathrm{~mm}$, 重点研究了激光功率及分层厚度 对复合材料性能的影响。

\section{2 复合材料构件性能评估方法}

火箭仪器支架在使用过程中受力情况比较复杂, 可能会受到拉伸、弯曲、冲击、振动等复杂应力的作 用及高温环境的影响, 同时, 支架还要与所要承载的 仪器以及舱壁进行可靠的装配与连接, 故在试验中需 采用多种测试手段综合表征复合材料的性能, 同时对 复合材料的装配性能也需要展开详细的研究。

样件的拉伸性能及弯曲性能采用万能力学试验 机(SANS CMT4304)进行测试, 测试条件及样件参 照国标 GBT 1040.1-2006、GBT 1040.2-2006 及 GBT 9341-2008; 样件的冲击强度测试采用摆锤试验机 (承德金和仪器制造有限公司, XJJ-50)进行测定, 相 关条件设定参照国标 GB/T1043.1-2008。

采用高低温电子万能试验机(Instron 5982)对复 合材料的高温力学性能进行了研究, 测试时, 首先 在室温下安装试样, 关闭炉门, 升温速度设定为 $4^{\circ} \mathrm{C}$ $/ \mathrm{min}$, 升温至设定温度后保温 $30 \mathrm{~min}$, 然后进行原
位拉伸试验, 测试温度设置为 $50^{\circ} \mathrm{C} 、 80^{\circ} \mathrm{C} 、 110^{\circ} \mathrm{C} 、$ $140^{\circ} \mathrm{C} 、 160^{\circ} \mathrm{C}$ 。采用动态热机械测试装置(DMA, 瑞士梅特勒 SDTA861e)测试试样的内损耗因子、储 能模量及损耗随着温度的变化情况, 测试采用双悬 臂弯曲模式, 振动频率 $1 \mathrm{HZ}$, 加载载荷 $10 \mathrm{~N}$ 。

对拉伸断面进行喷金, 利用扫描电子显微镜 (SEM,S-3000N,Hitachi)观察样件的内部形貌, 采用 溶液浸渍的方法测试样件的体积密度及孔隙率 ${ }^{[13]}$ 。

\section{2 试验结果分析}

\subsection{CF/PA12 复合粉末选择性激光烧结工艺优化}

由于选择性激光烧结的工艺特点, 其工艺条件 显著影响制件性能 ${ }^{[14-18]}$, 首先对选择性激光烧结制 备 CF/PA12 复合材料的工艺进行了优化。分别研究 了激光功率及分层厚度对制件强度的影响。固定分 层厚度 $0.15 \mathrm{~mm}$, 设定激光功率为 $8 \mathrm{~W} 、 10 \mathrm{~W} 、 12 \mathrm{~W}$ 、 $13 \mathrm{~W} 、 16 \mathrm{~W} 、 18 \mathrm{~W} 、 21 \mathrm{~W}$, 制件拉伸强度随着激光 功率的增加如图 3 所示, 在低激光功率下, 复合材 料制件强度较低, 甚至低于纯尼龙制件, 碳纤维的 增强效果主要体现在高激光功率区域。这主要是一 方面由于碳纤维具有较大的长径比, 在长度尺度上 与尼龙粉末不在一个数量级上, 从而造成复合粉末 的粉床密度较低, 内部存在大量的空隙(测试结果显 示 CF/PA12 的粉末床密度仅为 $0.474 \mathrm{~g} / \mathrm{cm}^{3}$ ), 从而 造成在选择性激光烧结过程中, 制件内部倾向于形 成多孔结构; 另一方面增强材料的加入也使得复合 粉末熔融时的粘度明显增加 ${ }^{[17]}$, 这就要求必须在较 高的激光功率下, 复合粉末的熔体粘度随着温度的 升高不断下降, 熔体流动性能增加, 从而制件的致 密度不断增加, 具有较高的强度, 但是当激光功率 较大时, 制件易发生尧曲及热老化, 制件的强度反 而下降，制件强度存在着一个最佳的激光功率。

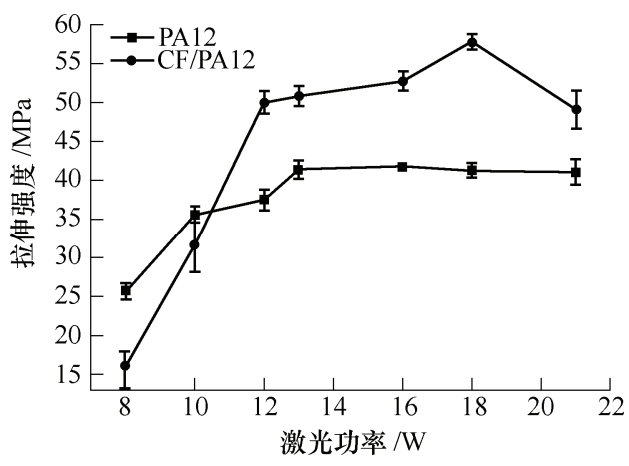

图 3 复合材料拉伸强度-激光功率图

此外, 分层厚度也是影响 SLS 制件强度的重要 工艺参数之一, 受到激光对粉末床穿透深度的影响, 
分层厚度越小, 在同样的激光能量密度情况下, 相 邻两层的结合越好, 制件的强度和表面质量更好, 但是分层厚度的选择受到粉末粒径的影响, 一般来 说, 分层厚度的取值必须大于粉末粒径, 其值过小 则会影响 SLS 的铺粉过程, 同时过小的分层厚度也 会造成加工时间过长等。在上部分试验的基础上, 选取较高的能量密度, 同时进一步降低分层厚度至 $0.125 \mathrm{~mm}$, 试验结果如图 4 所示, 从图中可以看出, 较小的分层厚度下, 复合材料制件均具有较高的强 度, 拉伸模量及弯曲性能也表现出类似的规律, 受 到粉末粒径的影响, 分层厚度无法进一步降低, 故 选定分层厚度为 $0.125 \mathrm{~mm}$, 激光功率 $18 \mathrm{~W}$ 作为 SLS 制备 CF/PA12 复合材料的最佳工艺条件, 以下样件 的制备均采用此工艺参数。

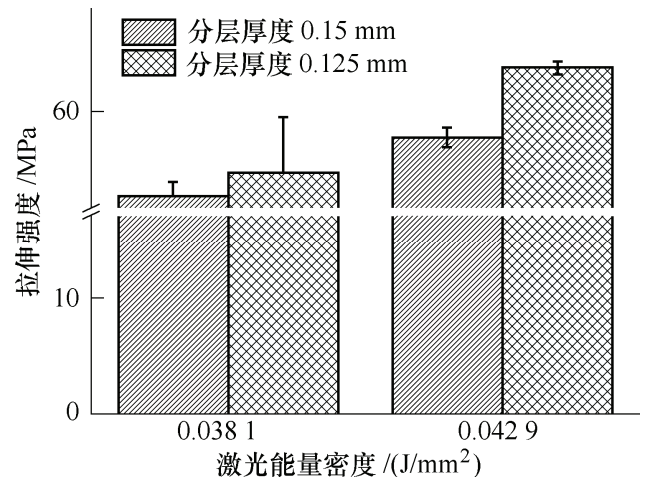

图 4 复合材料拉伸强度 VS 分层厚度

\section{2 复合材料试样性能研究}

飞行器仪器支架在使用过程中的受力情况比较 复杂, 包括拉伸、弯曲、冲击及振动等, 必须对材 料的性能进行全面的评估。复合材料的力学性能如 表 1 所示, 从表中可以看出, SLS 制备的 CF/PA12 复合材料的拉伸及弯曲性能远高于 PA12 的 SLS 制 件 ${ }^{[14]}$, 碳纤维起到明显的增强作用。但是其冲击强 度相对较低, 属于典型的脆性材料, 这主要是由于 SLS 制备过程中, 复合粉末的熔体粘度较大, 在没 有剪切力的作用下, 与纯 PA12 的 SLS 制件相比(如 图 5b 所示), 制件内部形成较多孔隙(如图 5a 所示), 这些孔隙的存在使材料的断裂伸长比与冲击强度大 幅下降; 另一方面, 这种情况下, 制件的密度较小, 测试结果显示, SLS 制备的 CF/PA12 复合材料的体 积密度仅为 $1.03 \mathrm{~g} / \mathrm{cm}^{3}$, 仅与纯尼龙的密度相当。

表 1 SLS 制备 CF/PA12 复合材料力学性能

\begin{tabular}{ccccccc}
\hline & $\begin{array}{c}\text { 拉伸强 } \\
\text { 度/MPa }\end{array}$ & $\begin{array}{c}\text { 弹性模 } \\
\text { 量/MPa }\end{array}$ & $\begin{array}{c}\text { 断裂伸 } \\
\text { 长比/\% }\end{array}$ & $\begin{array}{c}\text { 弯曲强 } \\
\text { 度/MPa }\end{array}$ & $\begin{array}{c}\text { 弯曲 } \\
\text { 模量 } \\
/ \mathrm{MPa}\end{array}$ & $\begin{array}{c}\text { 无缺口 } \\
\text { 冲击强度 } \\
/\left(\mathrm{J} / \mathrm{m}^{2}\right)\end{array}$ \\
\hline SLS-CF/PA12 & 63.8 & 6540 & 5.5 & 118.06 & 6016 & 9.46 \\
SLS-PA12 & 46 & 1602 & $36 \%$ & 46.3 & 1300 & 13.2 \\
\hline
\end{tabular}

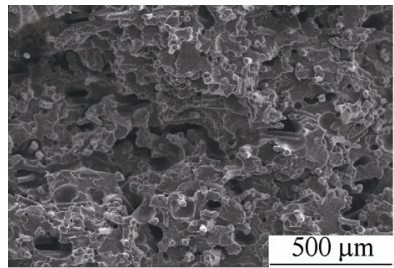

(a) $\mathrm{CF} / \mathrm{PA} 12$

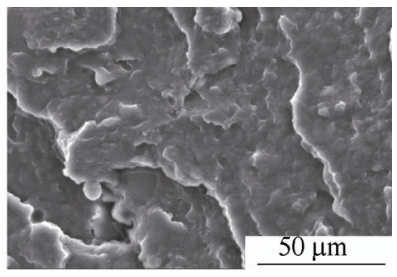

(b) 纯 PA12
图 5 SLS 制件微观形貌

飞行器仪器支架在使用过程中可能需要在较 高的环境温度中工作, 试验中采用在不同的温度 下进行拉伸强度的测试来表征材料的力学性能随 着温度的升高的变化情况, 测试过程如图 6a所示, 制件拉伸强度随温度的变化情况如图 $6 \mathrm{~b}$ 所示。从 图中可以看过, 虽然 PA12 的玻璃化转变温度较低 $\left(57{ }^{\circ} \mathrm{C}\right)$, 但是由于碳纤维的加入, 其复合材料的 力学性能并未在玻璃化转变温度以上发生突变, 复合材料的拉伸强度在 $100{ }^{\circ} \mathrm{C}$ 时维持在 $30 \mathrm{MPa}$ 以上, $150{ }^{\circ} \mathrm{C}$ 以上时力学性能较差, 这是由于此 时已经接近 PA12 的熔融温度, 材料呈现明显的高 弹态行为, 表现为模量和强度较低, 断裂伸长比 明显增大。

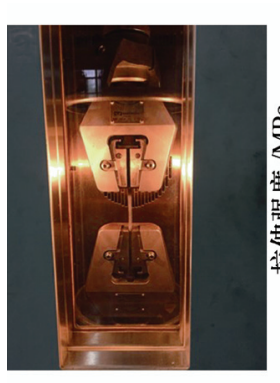

(a)

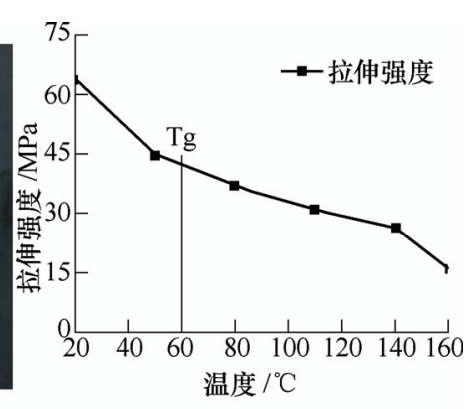

(b)
图 6 制件拉伸强度随温度的变化

DMA 测试的结果如图 7 所示, 从图中可以看 出复合材料的高温力学性能远远大于纯尼龙, 其储 能模量及损耗模量在高温下约为常温下的 $50 \%$ 左 右, 这与高温拉伸试验的结果也是相一致的。同时, DMA 的测试结果也包含了材料的损耗因子，损耗 因子又称阻尼因子或者损耗角正切, 这一数值显示 了粘弹性材料在动态变形下能量损耗的大小。损耗 因子随温度的变化情况如图 $7 \mathrm{c}$ 所示, 在 $25^{\circ} \mathrm{C} \sim$ $160^{\circ} \mathrm{C}$ 范围内, $\mathrm{CF} / \mathrm{PA} 12$ 复合材料的损耗因子分布在 $0.03 \sim 0.06$ 之间, 其值低于纯尼龙制件, 并且在尼 龙 12 的玻璃化转变温度附近 $\left(60^{\circ} \mathrm{C}\right)$ 达到最大值, 但 是远远大于金属材料的损耗因子, 这说明在振动环 境中, 高分子材料及其复合材料倾向于将一部分振 动能量转换为材料的内耗, 从而表现为具有更好的 减振性能。 


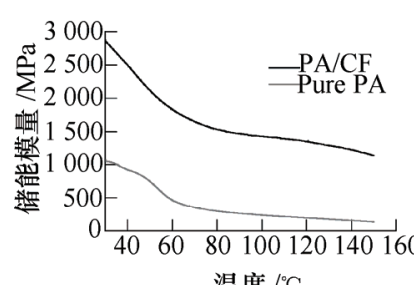

温度 $/{ }^{\circ} \mathrm{C}$

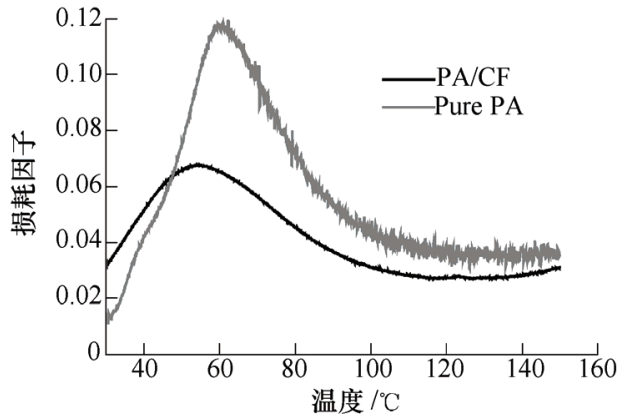

(c)

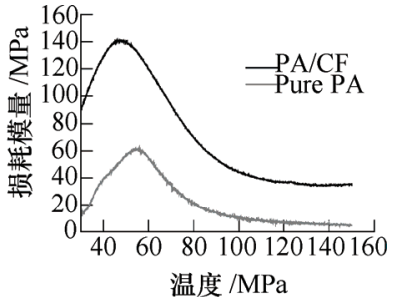

(b)
图 7 SLS 制备 CF/PA12 复合材料模量及 损耗因子随温度变化

\section{3 复合材料支架装配性能研究}

在实际使用过程中, 仪器支架需要与要承载的 仪器进行装配, 同时也需要安装在仪器舱基板上, 这就需要对 SLS 制备的复合材料制件探索合适的装 配方式。目前, 针对 SLS 制件的装配方式的研究较 少见诸报道, 不同于传统注塑工艺中, 螺纹套可通 过嵌件的方式固定在制件内, 对于 SLS 制件, 可进 行的连接方式包括粘结螺纹套、镶嵌螺母、法兰螺 母等方式, 在本部分, 分别采用这几种方式对复合 材料进行连接并测试了其螺纹拉脱力, 试验方案如 图 8 所示, 其中法兰螺母的装配采用在选取激光烧 结过程中打印出六边形孔, 与法兰螺母形成过盈配 合, 然后使用螺纹连接将上下两部分进行装配; 拉 脱力测试结果如图 9 所示, 从图中可以看出, 法兰 螺母的装配方式拉脱力最大, 其断裂方式为材料本 体破坏, 未发生螺纹滑脱等现象, 而粘结螺纹套和 镶嵌螺母装配体的破坏形式都是螺母滑脱, 说明对 于 3D 打印的 CF/PA12 复合材料制件, 通过法兰螺 母的形式进行装配是比较合适的。

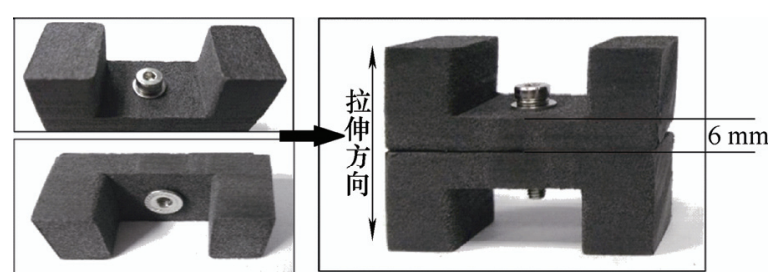

(a) (b)

图 8 拉脱力试验

选用法兰螺母的方式将复合材料进行连接并测 试了 M3-M8 的螺纹拉脱力, 其结果如图 10 所示,

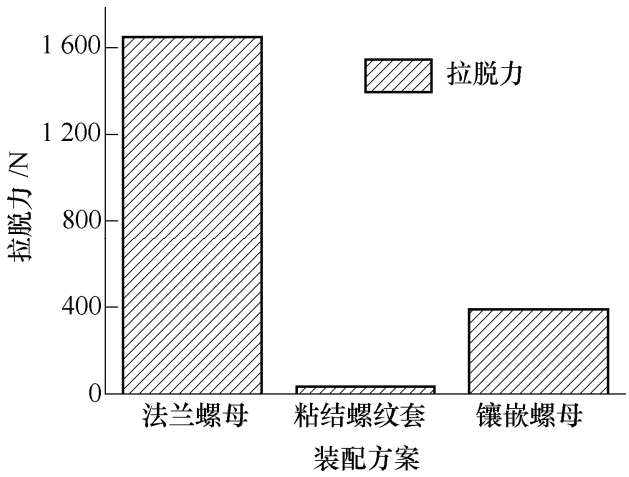

图 9 不同装配方式的拉脱力

拉脱力试验过程中破坏均发生在基体材料，当装配 螺纹孔较小时, 装配体的破坏形式表现为层间脱粘, 当装配螺纹孔较大时，装配体的破坏形式表现为弯 曲破坏。

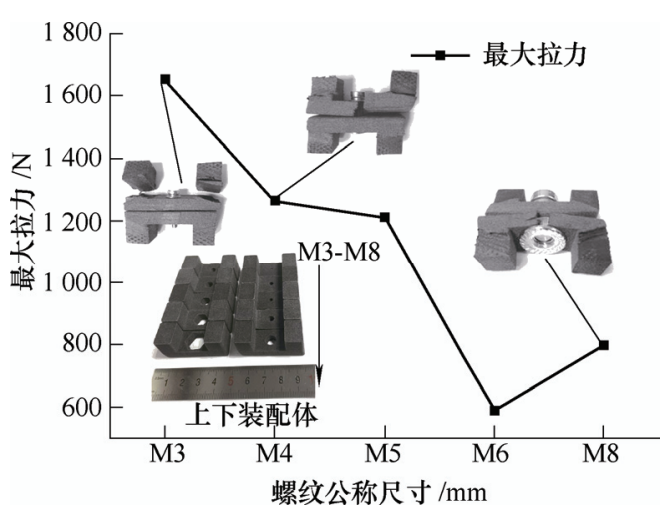

图 10 法兰螺母装配：M3-M6 拉脱力对比

此外，铆接也是一种在实际使用中应用较广的 装配形式, 可用于仪器支架与仪器舱基板的装配, 故用于仪器支架的复合材料必须能够进行铆接并在 铆接过程中无裂纹、破坏等产生。针对 SLS 制备的 复合材料样件也进行了铆接试验, 以观察其在铆接 过程中是否有裂纹及破坏发生, 试验结果如图 11 所示, 分别采用 $4 \mathrm{~mm}$ 和 $5 \mathrm{~mm}$ 抽芯铆钉、 $4 \mathrm{~mm}$ 厚 本体材料进行试验, 其结果表明, 材料可以进行铆 接，铆接后无裂纹产生。

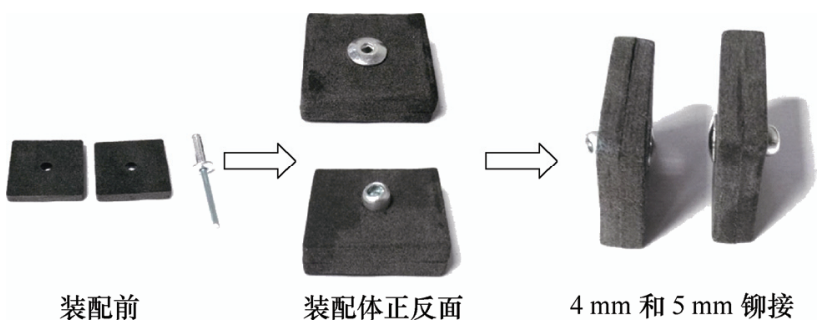

图 11 样件铆接过程

3 仪器支架试制及载荷试验

在性能测试的结果上, 对仪器支架进行了试制, 
所制备的支架如图 12a 所示, 支架重量为 $152 \mathrm{~g}$, 远 低于同体积的金属支架; 同时, 以能够承受 $2000 \mathrm{~N}$ 载荷为目标, 对支架进行了拓扑优化以进一步减轻 重量, 优化后的支架如图 $12 \mathrm{~b}$ 所示, 其重量仅为 81.6 $\mathrm{g}$ 。针对 $5 \mathrm{~kg}$ 级别载荷和 $10 \mathrm{~kg}$ 载荷, 按照 10 倍载 荷及 2 倍安全系数, 设置最大载荷为 $2000 \mathrm{~N}$, 试验 速度为 $0.5 \mathrm{~mm} / \mathrm{min}$, 进行了载荷试验, 考虑到火箭 升空时间较短, 故载荷保持时间设置较短。试验过 程及力-位移曲线如图 13 所示, 从图中可以看出, 当载荷在 $1000 \mathrm{~N}$ 以下时, 拓扑优化后的支架的位 移更小, 说明其具有更好的结构稳定性和刚度; 当 载荷继续增大时, 拓扑优化后的支架出现多次无法 保持载荷的情况, 这显示了拓扑优化之后的支架结 构稳定性在载荷较大时有所不足, 当载荷较大时, 可能局部发生了滑移。试验结束后卸除载荷, 支架 均无裂纹、无破坏发生, 说明该仪器支架承载 $10 \mathrm{~kg}$ 仪器时, 在两倍安全系数的前提下, 可以承受 10 倍的加速度。

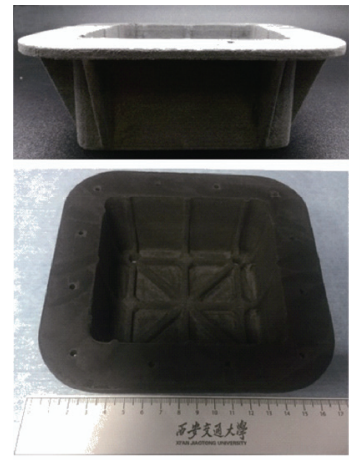

仪器支架 ( 重量 $152 \mathrm{~g}$ )

(a) 初始设计

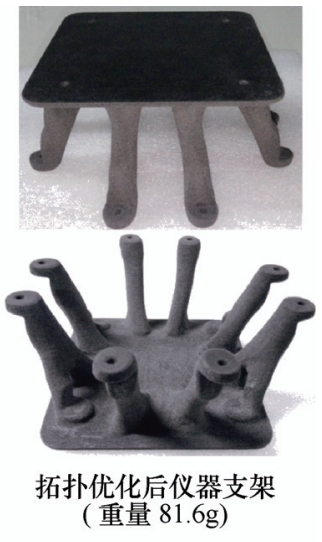

(b) 拓扑优化
图 12 仪器支架初始设计及拓扑优化结构
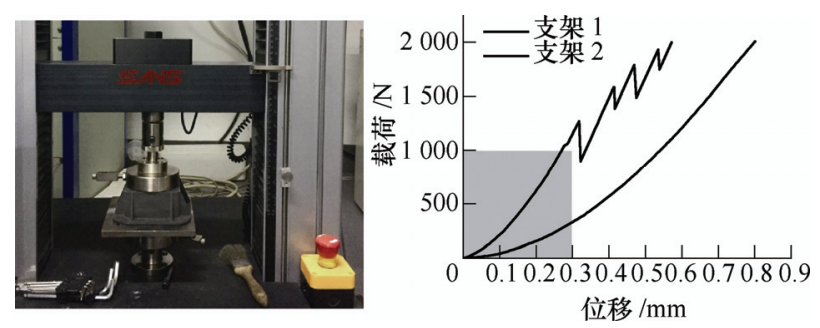

图 13 载荷试验过程及支架的力-位移曲线

\section{4 结论}

针对飞行器仪器支架的发展现状及使用要求, 本文提出使用选择性激光烧结(SLS)制备轻质复合 材料仪器支架以实现仪器支架的快速制备与结构优 化, 综合研究了 SLS 制备的短切碳纤维增强尼龙复 合材料的多种性能以验证其可行性。
(1) 测试结果表明, SLS 制备的 CF/PA12 相比较 纯尼龙制件具有更高的力学强度、模量及耐热性能, 与金属材料相比, 其具有较高的损耗因子及较低的 密度, 从而表现出更好的减振性能与较轻的质量。

(2) 针对 SLS 的工艺特点, 详细研究了所制备 的复合材料的装配方式, 评估了多种装配方式的螺 纹拉脱力和不同的螺纹孔装配体的破坏形式, 结果 表明, 采用法兰螺母是一种较为适宜的装配方式, 同时, 复合材料也可正常进行铆接, 从而保证了复 合材料仪器支架能够正常进行装配。

(3) 以一款火箭仪器支架为例, 采用 SLS 完成 了其快速制备; 同时, 对该仪器支架进行了拓扑优 化以进一步降低重量并成功制备了拓扑优化后的支 架, 优化后的支架重量仅为原重量的 $60 \%$, 试验结 果表明复合材料支架及其结构优化后的支架均能通 过 $2000 \mathrm{~N}$ 的载荷试验, 说明了采用 SLS 制备复合 材料在仪器支架轻量化、快速开发、拓扑优化等方 面具有明显的技术优势及在航空航天领域巨大的应 用空间。

\section{参 考 文 献}

[1] VASILIEV V V, RAZIN A F. Anisogrid conical adapters for commercial space application[C]//AIAA paper, Italy: 13th International Space Planes and Hypersonics Systems and Technologies, 2005: 16-20.

[2] 张阿舟, 楮德超, 姚起杭, 等. 实用振动工程 $[\mathrm{M}]$. 北 京: 航空工业出版社, 1997.

ZHANG Azhou, CHU Dechao, YAO Qihang, et al. Practical vibration engineering [M]. Beijing: Aviation Industry Press, 1997.

[3] 王海侨, 姜志国, 黄丽, 等. 阻尼材料研究进展 [J]. 高 分子通报, 2006(3): 24-30.

WANG Haiqiao, JIANG Zhiguo, HUANG Li, et al. Progress in damping materials research [J]. Polymer Bulletin, 2006(3): 24-30.

[4] 张忠明, 刘宏昭. 材料阻尼及阻尼材料的研究进展 [J]. 功能材料, 2001, 32(3): 227-230.

ZHANG zhongming, LIU Hongzhao. Progress of damping material and a damping material $[\mathrm{J}]$. Functional Materials, 2001, 32(3): 227-230.

[5] 蔡毅鹏, 王毅, 刘博, 等. 仪器舱整体减振支架二级减 振研究[J]. 导弹与航天运载技术, 2015(3): 53-56.

CAI Yipeng, WANG Yi, LIU Bo, et al. Study on secondary vibration reduction of instrument cabin integral damping bracket[J]. Missile and Space Vehicle Technology, 2015(3): 53-56.

[6] GOOdRIGE R D, TUCK C J, HAGUE R J M. Laser sintering of polyamides and other polymers[J]. Progress in 
Materials Science, 2012, 57(2): 229-267.

[7] SING S L, YEONG W Y. Direct selective laser sintering and melting of ceramics: A review[J]. Rapid Prototyping Journal, 2017, 23(3): 611-623.

[8] GHITA O, JAMES E, DAVIES R, et al. High temperature laser sintering (HT-LS): An investigation into mechanical properties and shrinkage characteristics of poly (ether ketone)(PEK) structures[J]. Materials \& Design, 2014, 61: 124-132.

[9] WANG Yuan, JAMES E, GHITA O R. Glass bead filled polyetherketone (PEK) composite by high temperature laser sintering (HT-LS)[J]. Materials \& Design, 2015, 83: 545-551.

[10] GUO Jiang, BAI Jiaming, LIU Kui, et al. Surface quality improvement of selective laser sintered polyamide 12 by precision grinding and magnetic field-assisted finishing $[\mathrm{J}]$. Materials \& Design, 2018, 138: 39-45.

[11] WU Jing, CHEN Hui, WU Qiong, et al. Surface modification of carbon fibers and the selective laser sintering of modified carbon fiber/nylon 12 composite powder[J]. Materials \& Design, 2017， 116: 253-260.

[12] 张正义, 陈英红, 戚方伟, 等. 固相剪切碾磨制备尼龙 12/多壁碳纳米管复合粉体及选择性激光烧结 3D 打印 [J]. 高分子材料科学与工程, 2017, 33(3): 122-127. ZHANG Zhengyi, CHEN Yinghong, QI Fangwei, et al. Preparation of nylon 12/multiwalled carbon nanotube composite powder by solid phase shear milling and selective laser sintering $3 \mathrm{D}$ printing $[\mathrm{J}]$. Polymer Science and Engineering, 2017, 33(3): 122-127.

[13] YAN Mengxue, TIAN Xiaoyong, PENG Gang, et al.
Hierarchically porous materials prepared by selective laser sintering[J]. Materials \& Design, 2017, 135: 62-68.

[14] VAN HOOREWEDER B, MOENS D, BOONEN R, et al. On the difference in material structure and fatigue properties of nylon specimens produced by injection molding and selective laser sintering[J]. Polymer Testing, 2013, 32(5): 972-981.

[15] CAULFIELD, MCHUGH P E, LOHFELD S. Dependence of mechanical properties of polyamide components on build parameters in the SLS process[J]. Journal of Materials Processing Technology, 2007, 182(1): 477-488.

[16] SENTHIKUMANRAN K, PANDEY P M, RAO P V M. Influence of building strategies on the accuracy of parts in selective laser sintering[J]. Materials \& Design, 2009, 30(8): 2946-2954.

[17] AKANDE S O, DALGAMO K W, MUNGUIA J, et al. Assessment of tests for use in process and quality control systems for selective laser sintering of polyamide powders[J]. Journal of Materials Processing Technology, 2016, 229: 549-561.

[18] YUAN Shangqin, BAI Jiaming, CHUA C K, et al. Material evaluation and process optimization of CNT-coated polymer powders for selective laser sintering[J]. Polymers, 2016, 8(10): 370.

作者简介: 晏梦雪, 女, 1985 年出生, 博士研究生。主要研究方向为复 合材料的选择性激光烧结。

E-mail: coolsnow@stu.xjtu.edu.cn

田小永(通信作者), 男, 1981 年出生, 博士, 副教授, 博士研究生导师。 主要研究方向为多材料、复合材料 $3 \mathrm{D}$ 打印(增材制造)技术及其应用。

E-mail: leoxyt@mail.xjtu.edu.cn 